\title{
The angiogenesis suppressor gene AKAP12 is under the epigenetic control of HDAC7 in endothelial cells
}

\author{
Andrei Turtoi - Denis Mottet $\cdot$ Nicolas Matheus $\cdot$ Bruno Dumont $\cdot$ \\ Paul Peixoto $\cdot$ Vincent Hennequière $\cdot$ Christophe Deroanne $\cdot$ Alain Colige $\cdot$ \\ Edwin De Pauw $\cdot$ Akeila Bellahcène $\cdot$ Vincent Castronovo
}

Received: 10 November 2011 / Accepted: 2 May 2012

(C) Springer Science+Business Media B.V. 2012

\begin{abstract}
Histone deacetylases (HDACs) are a family of 18 enzymes that deacetylate lysine residues of both histone and nonhistone proteins and to a large extent govern the process of angiogenesis. Previous studies have shown that specific inhibition of HDAC7 blocks angiogenesis both in vitro and in vivo. However, the underlying molecular mechanisms are not fully understood and hence preclude any meaningful development of suitable therapeutic modalities. The goal of the present study was to further the understanding of HDAC7 epigenetic control of angiogenesis in human endothelial cells using the proteomic approach. The underlying problem was approached through siRNA-medi-
\end{abstract}

Electronic supplementary material The online version of this article (doi:10.1007/s10456-012-9279-8) contains supplementary material, which is available to authorized users.

A. Turtoi $(\varangle) \cdot$ D. Mottet · N. Matheus - B. Dumont - P. Peixoto .

V. Hennequière $\cdot$ A. Bellahcène $\cdot$ V. Castronovo $(\square)$

Metastasis Research Laboratory, GIGA-Cancer, University Hospital,

University of Liège, Bat. B23, CHU Sart Tilman,

4000 Liège, Belgium

e-mail: a.turtoi@ulg.ac.be

V. Castronovo

e-mail: vcastronovo@ulg.ac.be

A. Turtoi · E. De Pauw

Laboratory of Mass Spectrometry, University of Liège,

Bat. B6C, 4000 Liège, Belgium

C. Deroanne $\cdot$ A. Colige

Laboratory of Connective Tissues Biology, GIGA-Cancer,

University Hospital, University of Liège, Bat. B23,

CHU Sart Tilman, 4000 Liège, Belgium ated gene-expression silencing of HDAC7 in human umbilical vein endothelial cells (HUVECs). To this end, HUVEC proteins were extracted and proteomically analyzed. The emphasis was placed on up-regulated proteins, as these may represent potential direct epigenetic targets of HDAC7. Among several proteins, A-kinase anchor protein 12 (AKAP12) was the most reproducibly up-regulated protein following HDAC7 depletion. This overexpression of AKAP12 was responsible for the inhibition of migration and tube formation in HDAC7-depleted HUVEC. Mechanistically, $\mathrm{H} 3$ histones associated with AKAP12 promoter were acetylated following the removal of HDAC7, leading to an increase in its mRNA and protein levels. AKAP12 is responsible for protein kinase $\mathrm{C}$ mediated phosphorylation of signal transducer and activator of transcription 3 (STAT3). Phosphorylated STAT3 increasingly binds to the chromatin and AKAP12 promoter and is necessary for maintaining the elevated levels of AKAP12 following HDAC7 knockdown. We demonstrated for the first time that AKAP12 tumor/angiogenesis suppressor gene is an epigenetic target of HDAC7, whose elevated levels lead to a negative regulation of HUVEC migration and inhibit formation of tube-like structures.

Keywords Vascular biology · Proteomics · Gene regulation $\cdot$ STAT3

$\begin{array}{ll}\text { Abbreviations } \\ \text { HATs } & \text { Histone acetyltransferases } \\ \text { HDACs } & \text { Histone deacetylases } \\ \text { HDACIs } & \text { HDAC inhibitors } \\ \text { HUVECs } & \text { Human umbilical vein endothelial cells } \\ \text { siRNA } & \text { Small interfering RNA } \\ \text { ECGS } & \text { Endothelial cell growth supplement } \\ \text { ChIP } & \text { Chromatin immunoprecipitation }\end{array}$




\section{Introduction}

Epigenetic modifications such as acetylation/deacetylation of histones play a key role in driving the expression of genes required for endothelial cell proliferation, migration and differentiation into new blood vessels (angiogenesis). Acetylation/deacetylation of histones is controlled by two families of enzymes with antagonized activity: histone acetyltransferases (HATs) and histone deacetylases (HDACs) [1]. HATs transfer acetyl groups to the epsilon amino group of evolutionarily conserved lysine residues located in the $\mathrm{N}$-termini of the core histones, which results in local expansion of chromatin and increased accessibility of regulatory proteins to DNA leading to gene transcription activation. Conversely, HDACs counteract the activity of HATs by catalyzing the removal of acetyl groups from lysine residues of histones, leading to chromatin condensation and gene transcription repression. Numerous studies have demonstrated that broad spectrum HDAC inhibitors (HDACIs) (effective for several HDAC family members) disturb the complex balance between the collective action of proangiogenic and antiangiogenic factors, which leads to inhibition of angiogenesis both in vivo and in vitro. Based on their potent antiangiogenic effects, several HDACIs are currently being investigated in clinical trials in cancer patients [2,3]. However, as such inhibitors are not specific to particular HDAC member, side effects have been reported with nearly all HDACIs. Therefore, targeting the most angiogenesis-relevant HDAC isoforms would substantially improve therapeutic efficacy and lower undesirable side effects of such drugs. In an effort to identify which HDAC is responsible for the antiangiogenic phenotype, we and others have recently demonstrated that the specific depletion of HDAC7 alters endothelial cell morphology, motility and prevents cell assembly in tube-like structures in vitro $[4,5]$. The mechanism by which HDAC7 depletion inhibits endothelial cell migration and disturbs cell morphology involves, at least in part, the stimulation of platelet-derived growth factor B, platelet-derived growth factor receptor $\beta$ and matrix metalloproteinase 10 expression. These findings were substantiated by further work reporting a role for HDAC7 in the maintenance of vascular integrity and regulation of angiogenesis, both in vivo and in vitro [5-7]. Consequently, HDAC7 may represent the most relevant target for inhibiting angiogenesis. However, several factors such as the similarity between the catalytic sites, the difficulty in obtaining purified, active enzymes and the relatively poor availability of useful crystal structures limit the development of pharmacological inhibitors specific to individual HDACs. Better and more complete understanding of the mechanisms of actions of HDAC7 in the regulation of angiogenesis should therefore contribute to the identification of new potential therapeutic targets that would bring about the desired antiangiogenic effect.
In the current study, HDAC7 silencing in human umbilical vein endothelial cells (HUVECs) combined with global proteomic analysis highlighted a repertoire of modulated proteins. As HDAC inhibition is typically permissive to gene expression, the primary focus was placed on the up-regulated proteins in HDAC7-depleted HUVECs. One of these was A-kinase anchor protein 12 (AKAP12), a member of the A-kinase-anchoring protein family, whose main function is to scaffold protein kinase A and C (PKA and $\mathrm{PKC}$ ) to distinct sites in the cell and in doing so, modulate their function [8]. Several findings suggest that AKAP12 has a tumor/angiogenesis suppressor role. For example, AKAP12 expression has been shown to downregulate the expression of proangiogenic genes such as vascular endothelial growth factor (VEGF) in tumor cells and interfere with angiogenesis and metastatic potential [9, 10]. Accordingly, AKAP12 has been found strongly diminished in various malignancies, including prostate, ovarian and breast tumors $[9,11,12]$. This suppression of AKAP12 was either sustained through deletions in the DNA or through epigenetic modifications such as DNA methylation [9]. Although the function of AKAP12 in tumor cells is being increasingly studied, little is known about the possible role of this protein in endothelial cells. In this study, we demonstrated for the first time that AKAP12 is under epigenetic control of HDAC7, whose depletion in HUVECs increased the histone $\mathrm{H} 3$ acetylation levels at the AKAP12 promoter and consequently induced its expression at both mRNA and protein levels. This upregulation of AKAP12 is responsible for the inhibition of endothelial cell migration and differentiation into capillary tube-like structures, effects observed in the absence of HDAC7. Mechanistically, we showed that AKAP12 controls the phosphorylation of signal transducer and activator of transcription 3 (STAT3), a key post-translational modification required for its transcriptional activation. Interestingly, we noticed that STAT3 is also essential for maintaining augmented levels of AKAP12, suggesting a positive regulatory control loop between AKAP12 and STAT3. In summary, this study shows a novel molecular mechanism of action of HDAC7 during angiogenesis involving a new player-AKAP12.

\section{Materials and methods}

Unless otherwise specified, all reagents used were obtained from Sigma Aldrich (St. Louis, MO, USA) and were of the highest grade available. An outline of the study design (Figure S1) and further description of all materials and methods are presented in the Supplemental Data section. All experiments were at least performed as independent triplicate manipulations (biological replicates). 
Cell culture and siRNA mediated knockdown of HDAC7, AKAP12 and STAT3

HUVECs were cultured in gelatin precoated cell culture dishes $\left(55 \mathrm{~cm}^{2}\right.$ ). $2 \mathrm{~mL} 0.2 \%$ gelatin (Sigma Aldrich, St. Louis, MO, USA) was spread in the dishes and incubated for 15 min at RT. Surplus gelatin was removed and the coated dishes were washed twice with PBS (Invitrogen, Carlsbad, CA, USA). The cells were isolated from human umbilical cord as described previously [13] and maintained in enriched MCDB-131 culture medium (Gibco, Carlsbad, CA, USA) supplemented with $20 \%$ fetal bovine serum (FBS), $2 \mathrm{mM} \mathrm{L}_{\text {- }}$ glutamine, $1 \%$ penicillin-streptomycin, heparin (final concentration $50 \mu \mathrm{g} / \mathrm{mL}$ ) and $50 \mu \mathrm{g} / \mathrm{mL}$ endothelial cell growth supplement (ECGS) (Becton Dickson, Franklin Lakes, NJ, USA). $1.2 \times 10^{6}$ HUVECs (passage 7) were seeded the day before transfection in order to reach 70-80 \% of confluence. The cells were then transfected with small interfering RNA (siRNA) at a final concentration of $60 \mathrm{nM}$ using the GeneTrans II Kit (MobiTec, Göttingen, Germany) according to the manufacturer's instructions. siRNA specific to HDAC7 ([\#1] 5'-GGA-CAA-GAG-CAA-GCG-AAG-U$3^{\prime}$, [\#2] 5'-GGC-UGG-AAA-CAG-AAA-CCC-A-3' and [\#3] 5'-CCU-GAA-GCU-GCG-CUA-UAA-G-3'), AKAP12 (5'-UCU-GCA-GAA-UCU-CCG-ACU-AUU-3') and STAT3 ( $5^{\prime}$-AAA-GUC-AGG-UUG-CUG-GUC-AAA-UU-3') were used to silence the corresponding target genes. Scrambled HDAC7 siRNA (HDAC7 scrRNA) (5'-CCU-GUU-CUCGUU-CGC-UUC-A-3') was used as a negative control for HDAC7 knockout conditions, whereas GL3 luciferase siRNA ( $5^{\prime}$-CUU-ACG-CUG-AGU-ACU-UCG-A-3') was used for all co-transfection experiments. In addition, cells were also mock transfected with the same kit but without any siRNA (no siRNA condition). All HDAC7, AKAP12 and STAT3 siRNA as well as all scrambled siRNA were obtained from Eurogentec (Liège, Belgium). Four hours after transfection, the cells were washed twice with PBS and serum-free culture medium. Following this, the cells were incubated in enriched medium for additional time (resulting in total of $48 \mathrm{~h}$ ) and subsequently used for proteomic analysis and various assays.

Tube-like structure formation assay

To induce a network of tube-like structures, HUVECs were cultured between two gels of collagen as described previously [14]. In short, 6-well plates were coated with a 0.4mm-thick collagen gel (DMEM medium [Gibco] supplemented with $1 \mathrm{mg} / \mathrm{mL}$ purified rat tail type I collagen, $\mathrm{pH}$ 7.3) and allowed to polymerize for $15 \mathrm{~min}$ at $37^{\circ} \mathrm{C}$. Cotransfected HUVECs $\left(4 \times 10^{5} /\right.$ well) (same conditions as for the migration assay) were allowed to attach over a period of $6 \mathrm{~h}$. After spreading, the cells were covered with a second layer of collagen. Enriched MCDB-131 culture medium was added after the gel polymerization. After $24 \mathrm{~h}$, pictures were taken with a phase-contrast microscope (EVOSfl) and the density of the tube network was evaluated using the ImageJ software (NIH, Washington, D.C., USA).

\section{Cell migration assay}

To assess the impact of AKAP12 on endothelial cell migration, cotransfection experiments were conducted: HDAC7/GL3, AKAP12/GL3, HDAC7/AKAP12 and GL3/ GL3. Each siRNA was used at a concentration of $30 \mu \mathrm{M}$. Transfected cells were collected $24 \mathrm{~h}$ after transfection. Two-chambered silicon inserts for wound-healing assay (Ibidi, Martinsried, Germany) were placed in the middle of the gelatin-coated 6-well plates $\left(9.6 \mathrm{~cm}^{2} /\right.$ well). 6000 HUVECs in $70 \mu \mathrm{L}$ of the enriched MCDB-131 medium were seeded in each of the two separate chambers. Following an additional $24 \mathrm{~h}$, the inserts were removed and the cells were washed twice with $2 \mathrm{~mL}$ MCDB-131 medium. Migration assay was then performed in $2 \mathrm{~mL}$ enriched medium. The migration of the cells towards the wound was followed by phase-contrast microscopy (EVOSfl, Advanced Microscopy Group, Bothell, WA, USA) and the experiments were stopped before the complete closure of the wound in the control condition $(\sim 5 \mathrm{~h})$. Following the migration, measurements of the wound closure were performed and the values were converted to percentages accordingly.

\section{Chromatin immunoprecipitation}

The assessment of acetylation level of histones $\mathrm{H} 3$ on human AKAP12 promoter as well as the binding of STAT3 on ICAM1 and AKAP12 promoters was performed using a LowCell chromatin immunoprecipitation (ChIP) kit (Diagenode, Liège, Belgium) according to the manufacturer's instructions. In short, $1 \times 10^{6}$ cells were disrupted for $2 \times 10$ min with a Diagenode Bioruptor (30 s on, $30 \mathrm{~s}$ off; high setting). The isolated chromatin equivalent of $1 \times 10^{6}$ cells was immunoprecipitated with either $3 \mu \mathrm{g}$ pan-acetyl histone H3 (Millipore, Billerica, MA, USA; Cat. \# 06-599), $6 \mu \mathrm{g}$ STAT3 (Santa Cruz Biotechnology, USA; Cat.No.: SC-482) or $6 \mu \mathrm{g}$ rabbit IgG (Zymed, San Fancisco, CA, USA; Cat. \#02-6102). After reversal of crosslinking, the immunoprecipitated DNA was purified by a regular DNA extraction protocol and analyzed employing RT-qPCR with the SYBR-Green Supermix (Roche, Mannheim, Germany) and ABI-7300 cycler. The PCR conditions were $10 \mathrm{~min}$ at $95{ }^{\circ} \mathrm{C}$ followed by 40 cycles of $10 \mathrm{~s}$ at $95^{\circ} \mathrm{C}$, $30 \mathrm{~s}$ at $60{ }^{\circ} \mathrm{C}$ and $30 \mathrm{~s}$ at $72{ }^{\circ} \mathrm{C}$. The following, previously published, primers were used [15]: promoter AKAP12 (5'- 
TGC-CAA-CCG-TCT-TGA-AGC-AA- $3^{\prime}[\mathrm{F}]$ and $5^{\prime}$-AAGGGC-CAT-GGT-GCT-GAG-TC- $3^{\prime}$ [R]), promoter ICAM1 (5'-CCT-GGA-GTC-TCA-GTT-TAC-CGC-TTT-3' $[\mathrm{F}]$ and 5'-GCT-GCA-GTT-ATT-TCC-GGA-CTG-ACA-3' $[$ R]).

Inhibition of JAK2, PKA and PKC

$2 \times 10^{5}$ HUVECs were plated in 6-well plates and transfected as described above. After $24 \mathrm{~h}$, cells were treated by replacing the medium with either $20 \mu \mathrm{M}$ AG 490 JAK2 inhibitor, $5 \mu \mathrm{M} \mathrm{H} 89$ PKA inhibitor or 1 (and 0.5) $\mu \mathrm{M}$ Gö6976 PKC inhibitor (Merck-Calbiochem, Darmstadt, Germany) dissolved in DMSO. Medium was removed after $16 \mathrm{~h}$ and the cells were washed twice with PBS.

\section{Statistics}

Whenever indicated, statistical evaluation was performed using a two-sided, unpaired Student's $t$-test, assuming equal variances (Excel, Microsoft, Redmond, WA, USA). The employment of the $t$-test was justified because the data followed normal distribution (assessed in separate test, data not shown).

\section{Results}

HDAC7-depletion-induced proteomic alterations in HUVECs

The proteomic investigation of HDAC7-depleted HUVECs established a repertoire of 15 up-regulated proteins that are potentially epigenetically regulated (Table S1). A protein was considered to be up-regulated if its expression ratio was at least 1.5 -fold higher $(p \geq 0.95)$ in the HDAC7 siRNA condition compared to mock-transfected HUVECs (no siRNA). Further criteria were that the given overexpression had to be observable in at least two out of three biological replicates and the protein in question should not be significantly modulated $(p<0.95)$ between the mockand HDAC7-scrRNA-transfected HUVECs. Some proteins were detected only when HDAC7 was absent (only in the siRNA samples). As these proteins were not detected under other conditions (mock- or HDAC7-scrRNA-transfected conditions), no values for the fold expression could be calculated. Such proteins were considered to be up-regulated and if the observation persisted in at least two out of three replicates they were included in Table S1. The downregulated proteins were not of primary interest in the present work as they represent indirect HDAC7 epigenetic targets. However, the significantly down-regulated proteins found in the current study are outlined in the Supplemental Data (Table S1). One down-regulated protein, namely dihydropyrimidinase related protein 3 , has been selected and further validated using WB and RT-qPCR (Supplemental Data, Figure S2).

AKAP12 protein fulfilled the outlined selection criteria in all three biological replicates. As AKAP12 gene is transcribed into two different transcript variants, both were quantified at the gene expression level. The up-regulation of AKAP12 expression following HDAC7 depletion was confirmed using three different siRNAs at both protein (Western blot) and mRNA (RT-qPCR) levels (Fig. 1a, b).

Induction of AKAP12 expression contributes to HDAC7 depletion-mediated inhibition of endothelial cell migration and tube-like structure formation

We previously demonstrated that HDAC7 depletion in HUVECs leads to impediment of migration and inhibition of tube-like structure formation [4]. In order to examine

A

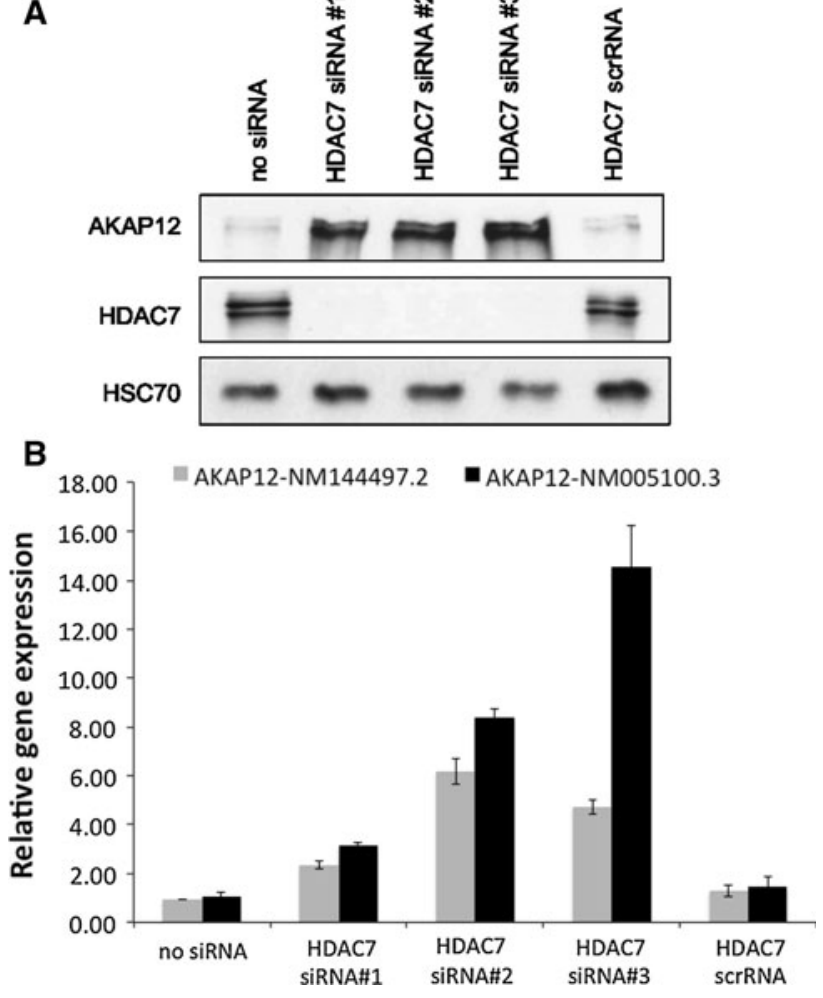

Fig. 1 HDAC7 silencing modulates AKAP12 gene- and proteinexpression levels. a Western blot analysis of AKAP12 protein expression in HUVECs following the depletion of HDAC7. The HSC70 protein was used for normalization of protein loading. b Gene-expression modulation of AKAP12 measured with RT-qPCR in HDAC7-depleted HUVECs. Both isoforms of AKAP12 are modulated at the protein- and gene-expression level. The HDAC7 siRNA\#1-3 are described in the "Materials and methods" section. Error bars indicate standard deviation of means where $n=3$ independent biological replicates 


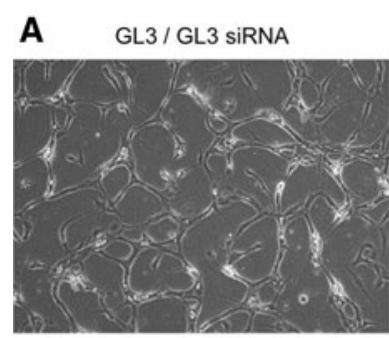

HDAC7 / GL3 siRNA
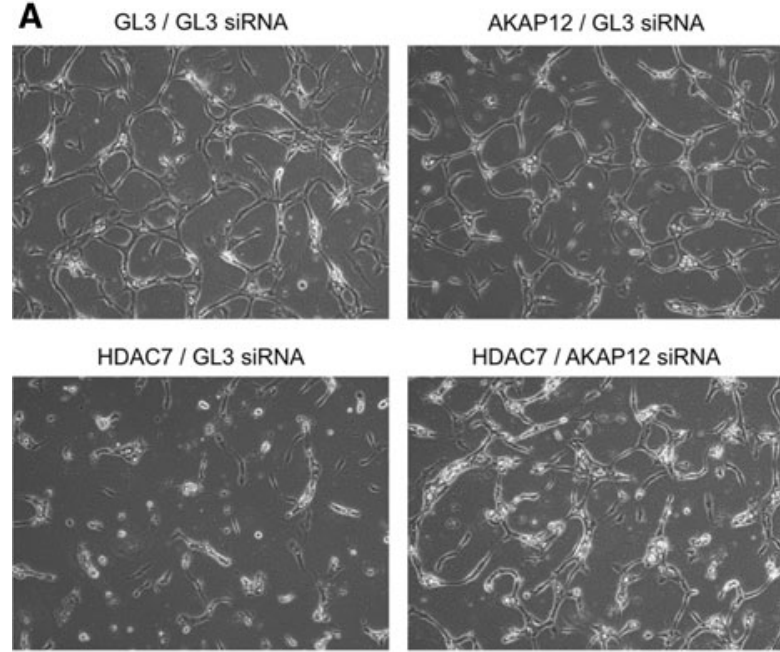

HDAC7 / AKAP12 siRNA

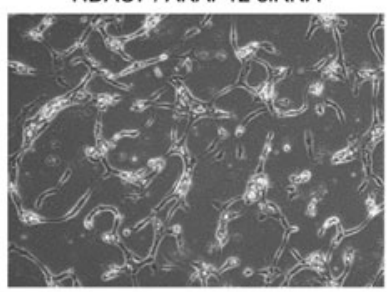

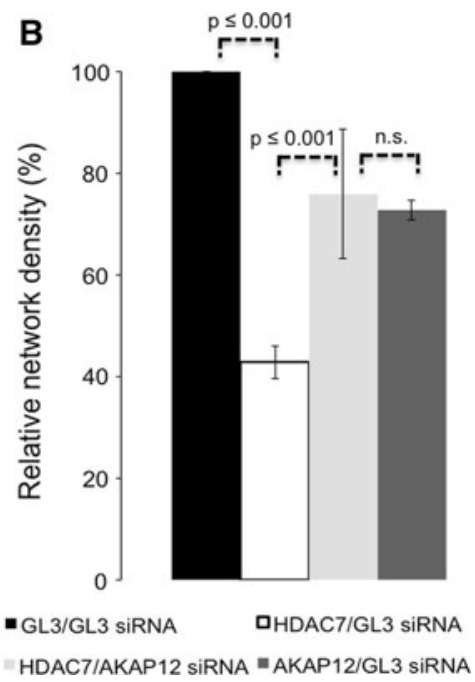

HUVECs were used as a reference (set to $100 \%$ ) for quantification of network density. A representative WB assessing the depletion of HDAC7 and AKAP12 is shown in the Fig. 3c. Error bars indicate standard deviation of means where $n=3$ independent biological replicates

HDAC7 depletion increases AKAP12 expression through epigenetic modification at its corresponding promoter and requires STAT3 transcription factor

Seeking to understand how HDAC7 controls AKAP12 expression, we first assessed the presence of acetylated histone $\mathrm{H} 3$ at the AKAP12 promoter in HDAC7-depleted HUVECs. The ChIP analysis demonstrated a significant increase in acetylated histone $\mathrm{H} 3$ on the AKAP12 promoter following HDAC7 depletion in HUVEC (Fig. 4a). Although these data demonstrated that the removal of HDAC7 leads to increased acetylation of the histone $\mathrm{H} 3$ on the AKAP12 gene promoter, which in turn increases its mRNA and protein levels, the transcription itself is dependent on suitable transcription factors. According to the previously published ChIP data [15, 16], AKAP12 gene has been demonstrated as a direct target of STAT3 transcription factor in lung epithelial cells. Based on this information, we hypothesized that STAT3 may positively control expression of AKAP12 in HUVECs. Indeed, this assumption was strengthened by the subsequent ChIP analysis where increased STAT3 binding to the AKAP12 promoter was detectable following the suppression of HDAC7 (Fig. 4b).

STAT3 is a transcription factor that undergoes a series of post-translational modifications (PTMs) that are decisive for its activity and subcellular localization [17]. Prior to assuming its gene-modulation role, we first focused on characterization of STAT3 PTM following the HDAC7 knockdown in HUVECs. We examined the phosphorylated 
Fig. 3 Impairment of HUVEC migration following HDAC7 depletion is dependent on AKAP12 protein expression. $\mathbf{a}$ and $\mathbf{b}$ Inhibition of HDAC7 alone induces a $\sim 50 \%$ decrease in HUVEC migration, whereas cells cotransfected with siAKAP12 and siHDAC7 reduce the migration by only $\sim 25 \%$. The latter value is significantly different from both nontransfected and siHDAC7 transfected cells (two-sided $t$ test). AKAP12 depletion alone does not affect migration in HUVECs. Nontransfected cells (Nosi) were set to $100 \%$ as a reference. c Depletion of both HDAC7 and AKAP12 was monitored using the WB. Error bars indicate standard deviation of means where $\mathrm{n}=6$ independent biological replicates
A

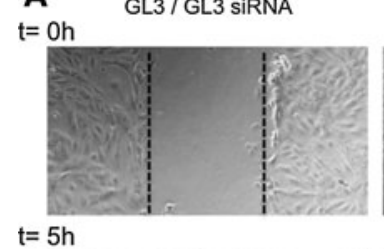

AKAP12 / GL3 siRNA
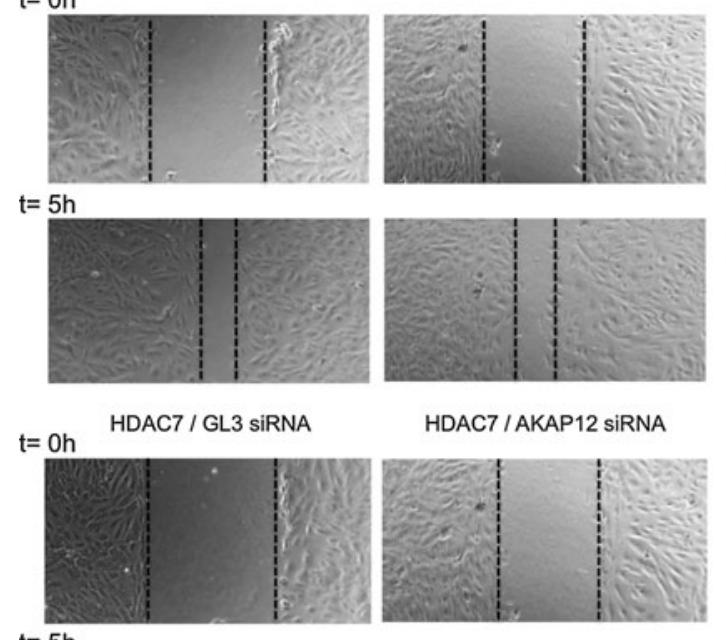

HDAC7 / AKAP12 SIRNA
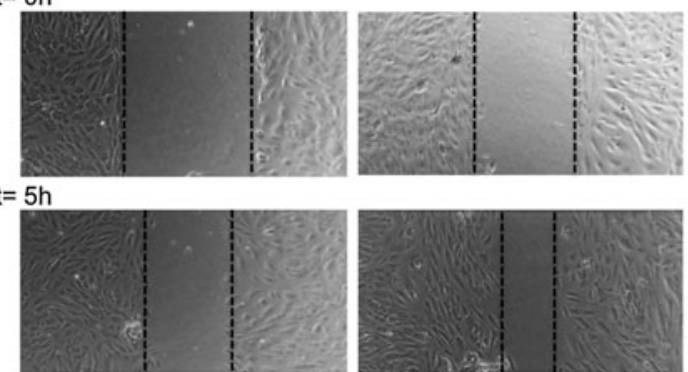

B

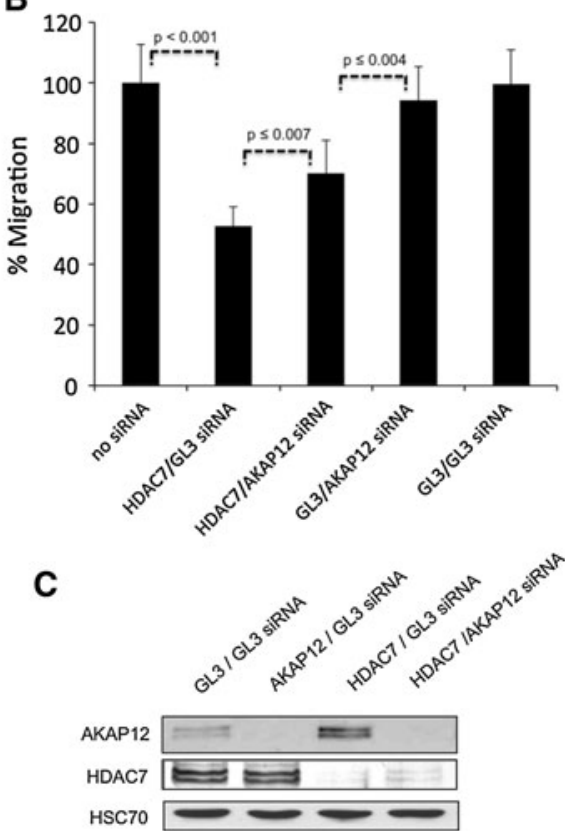

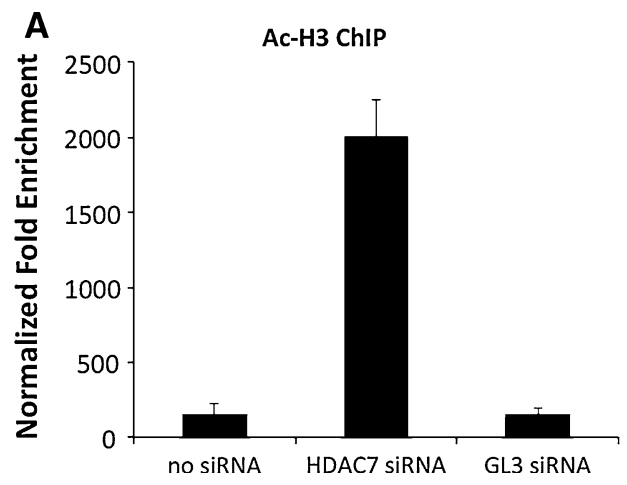

Fig. 4 ChIP analysis of a acetylated histone H3 and b STAT3 on AKAP12 promoter. Depletion of HDAC7 in HUVEC increases both the levels of acetylated histone H3 and STAT3 on the AKAP12 promoter. Results are expressed as normalized fold enrichment

status of two different amino acid residues (Y705 and S727) by Western blot. The results indicated an increased level of phospho-Y705 in the absence of HDAC7. No changes in the phospho-S727 status or in the total level of this protein were detectable (Fig. 5a). As the phosphorylation of STAT3 at Y705 is required for its nuclear translocation and its DNA binding, we next examined the exact subcellular localization of STAT3 pY705 following the silencing of HDAC7. For this purpose, a subcellular fractionation assay followed by a Western blot analysis was performed, revealing that in the absence of HDAC7 STAT3 pY705 is more abundant in the nuclear compartment (Fig. 5b). Immunofluorescence staining of STAT3 pY705 (Fig. 5c) upon the depletion of HDAC7 in HUVEC confirmed these findings.

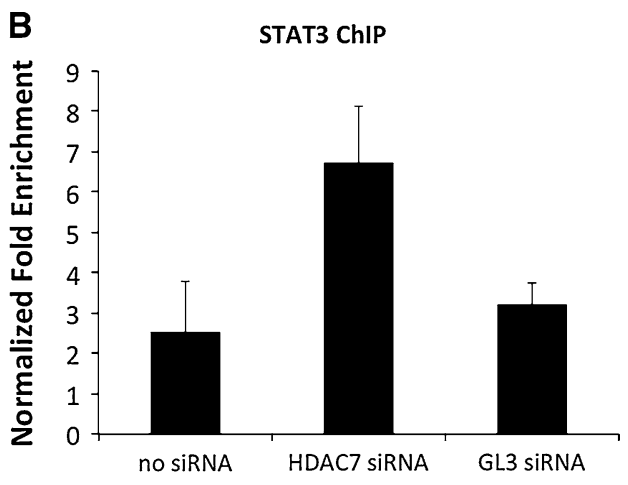

values, where the normalizator is the isotype $\operatorname{IgG}$ antibody. Error bars indicate standard deviation of means where $n=3$ independent biological replicates

To further characterize the intranuclear localization of STAT3 pY705, we also performed a protein fractionation, subdividing the proteome into cytoplasmic, soluble nuclear and chromatin-bound fractions. We observed that STAT3 pY705 is more abundant in the chromatin-enriched fraction following the depletion of HDAC7 (Fig. 5d). Collectively, the data indicate that depletion of HDAC7 in HUVECs causes increased phosphorylation of STAT3 on Y705. This phosphorylated form of STAT3 is predominately found in the nucleus of HUVECs, bound to the chromatin.

In order to conclusively determine whether STAT3 is involved in the AKAP12 expression in HDAC7-depleted cells, we cosilenced the expression of both HDAC7 and STAT3. Cosuppression of HDAC7 and STAT3 markedly curbs the augmentation of AKAP12 levels induced through 


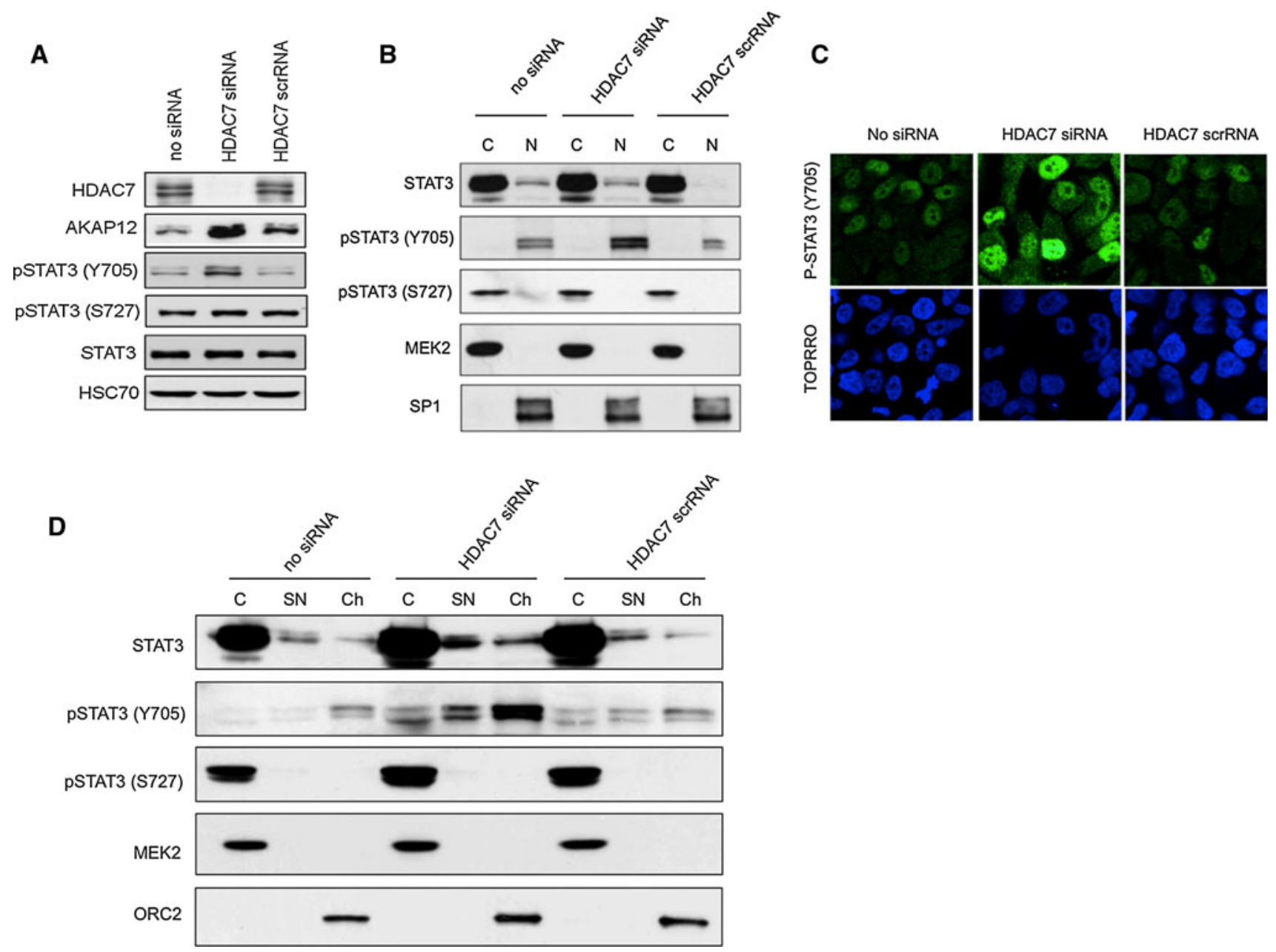

Fig. 5 Depletion of HDAC7 induces phosphorylation of STAT3 in the nucleus of HUVECs and increased binding to chromatin. a HDAC7 silencing induces phosphorylation of STAT3 on Y705 in HUVECs. In contrast, the S727 phosphorylation as well as the total levels of STAT3 are unaffected. b STAT3 pY705 is predominantly found in the nuclear fraction. c Immunofluorescence staining of STAT3 pY705 demonstrates the overexpression of this protein in the

HDAC7 depletion. The findings suggested that STAT3 positively regulates AKAP12 protein expression in HUVECs (Fig. 6) and that this transcription factor is in fact essential for maintaining elevated AKAP12 levels upon HDAC7 silencing.

ICAM1 is a further angiogenesis-relevant STAT3 target in HUVEC

Following the finding that STAT3 is activated upon HDAC7 suppression in HUVEC, we sought to investigate whether other angiogenesis relevant STAT3-target genes may be modulated as well. For this purpose we have examined the existing literature and have preselected a group of 10 genes (all STAT3 targets and relevant for angiogenesis) and tested their putative modulation using nucleus of HUVECs upon removal of HDAC7. d HDAC7 silencing induces accumulation of STAT3 pY705 in the chromatin-enriched fraction of HUVEC. The following abbreviations were used: b $C$ cytoplasmic fraction, $N$ nuclear fraction; d $S$ soluble cytoplasmic proteins, $S N$ soluble nuclear proteins, $C h$ chromatin-bound proteins; representative images of one out of three independent biological replicates

RT-qPCR $48 \mathrm{~h}$ after the HDAC7 depletion in HUVEC (Fig. 7a). Of the selected genes, ICAM1 tested positive displaying significant up-regulation upon HDAC7 depletion in HUVEC. The modulation of ICAM1 gene expression is separately shown in the Fig. 7b. This up-regulation was confirmed on the protein level too (Fig. 7c). Following the HDAC7 silencing, STAT3 directed ChIP analysis has demonstrated the increased binding of STAT3 on the ICAM1 promoter, identifying a further angiogenesis relevant STAT3 target in endothelial cells (Fig. 7d).

Phosphorylation of STAT3 in HDAC7 depleted HUVEC is PKC dependent

HDAC7 depletion leads to an increase in the phosphorylation of STAT3, suggesting that a kinase is probably 


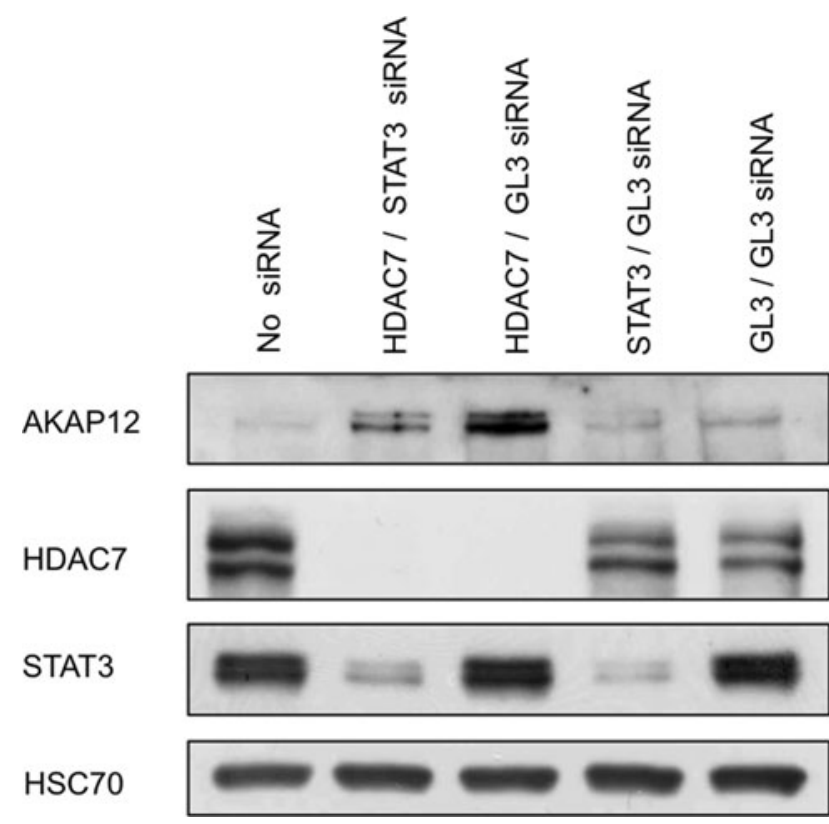

Fig. 6 STAT3 controls the expression of AKAP12 in HUVECs. Depletion of HDAC7 in HUVECs leads to augmented levels of AKAP12, which is reversible when STAT3 is silenced; representative images of one out of three independent biological replicates involved. Accordingly, kinases such as JAK2 have been previously reported as implicated in phosphorylating the Y705 residue. PKA and PKC also showed the ability to phosphorylate STAT3 $[18,19]$. Both kinases were particularly relevant because AKAP12 is known to participate in their scaffolding. To identify which kinase phosphorylates STAT3 on Y705, we used chemical compounds to inhibit JAK, PKA and PKC kinases and assessed the modulation of STAT3 pY705 following the depletion of HDAC7. Neither the PKA inhibitor (H-89) nor the JAK inhibitor (AG490) had an effect on STAT3 pY705 in the absence of HDAC7 (Fig. 8a). However, treatment of HDAC7-depleted HUVECs with Gö6976, an inhibitor of PKC (with high affinity mainly for isoforms $\alpha / \beta$ ), effectively inhibited the phosphorylation of STAT3 Y705 in dose-dependent fashion (Fig. 8b).

AKAP12 is required for phosphorylation of STAT3 Y705 in HDAC7-depleted HUVEC

As described above, AKAP12 is a known scaffolding partner of PKC [10] and therefore may be also directly involved in the STAT3 phosphorylation event. Cotransfection experiments
Fig. 7 a RT-qPCR screening of several STAT3 target genes known to be involved in angiogenesis. ICAM1 gene expression was found modulated upon HDAC7 depletion in HUVEC. b RTqPCR data shows a fourfold increase in ICAM1 mRNA levels following HDAC7 suppression in HUVEC. c ICAM1 up-regulation was confirmed at the protein level using Western blot analysis. d ChIP analysis demonstrated the increased presence of STAT3 on the ICAM1 promoter, supporting the STAT3-mediated regulation. Error bars indicate standard deviation of means where $n=3$ independent biological replicates
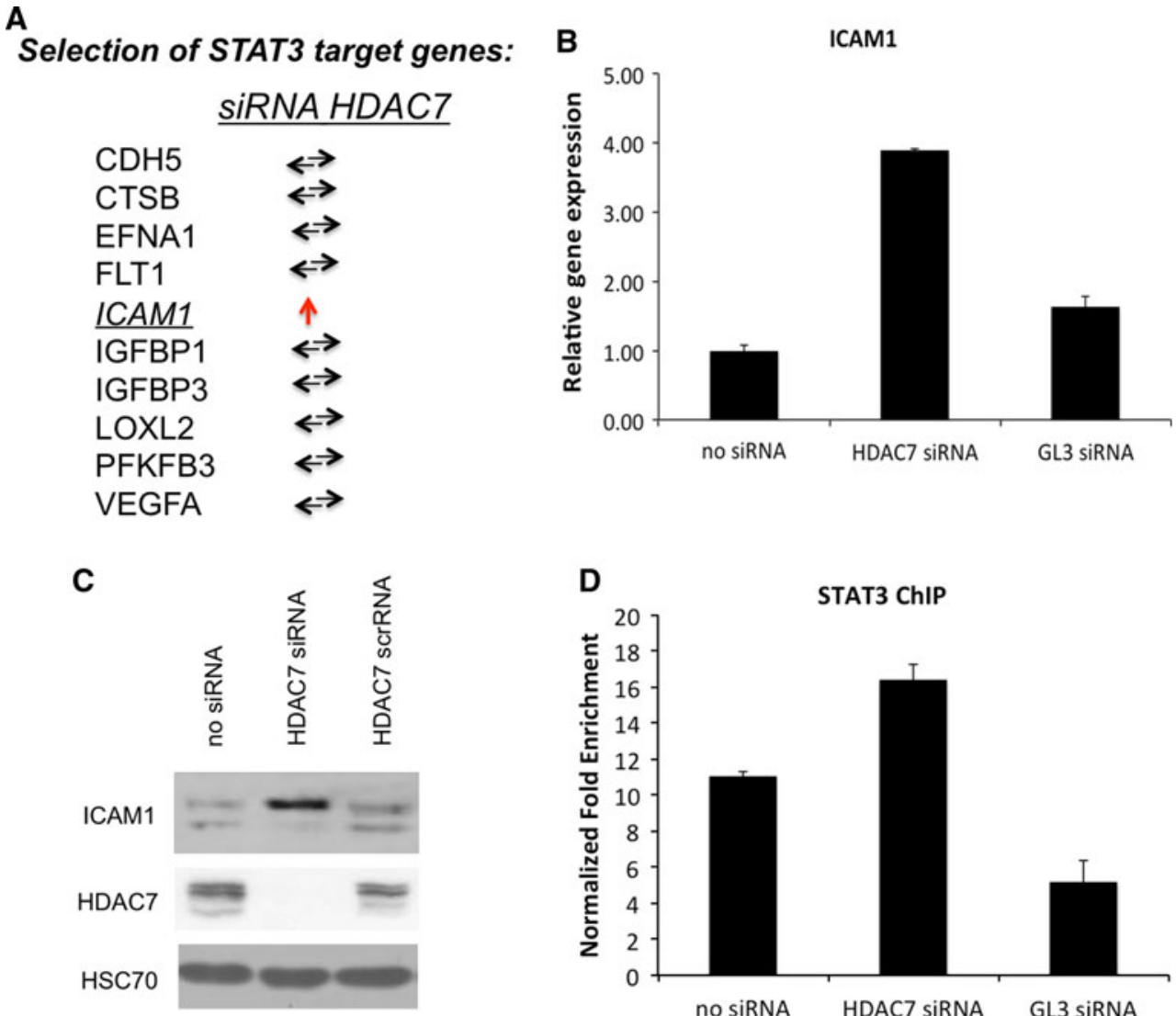

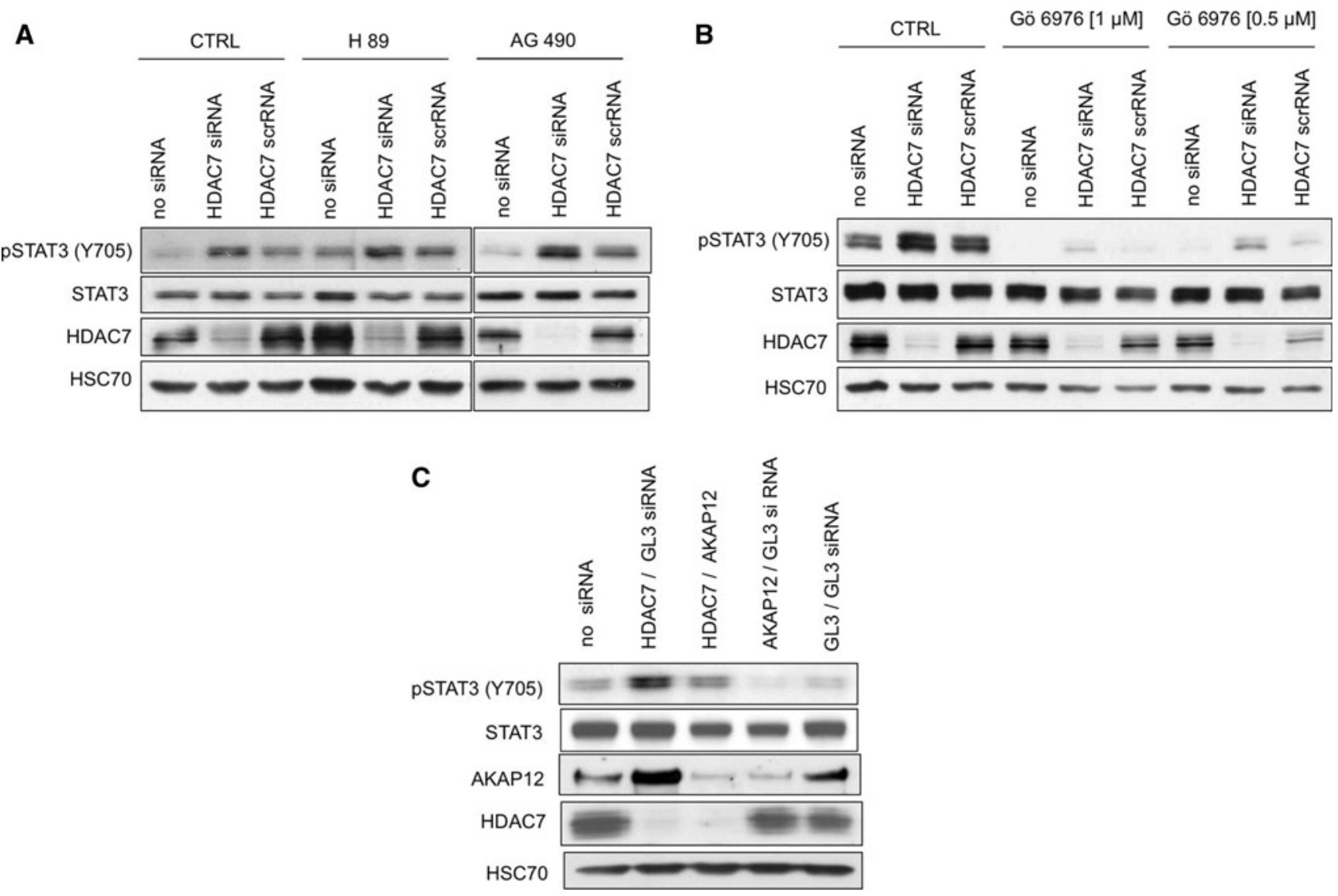

Fig. 8 PKC controls the phosphorylation of STAT3 on Y705 and this process is dependent on AKAP12 expression. a Inhibition of PKA (H 89) and JAK2 (AG 490) kinases does not influence the phosphorylation of STAT3 on Y705 following the depletion of HDAC7 in HUVECs. b The inhibition of PKC through inhibitor Gö6976 suppresses the levels of STAT3 pY705 in a dose-dependent manner. c HDAC7 depletion-induced phosphorylation of STAT3 Y705 in HUVECs. Cosuppression of HDAC7 and AKAP12 abolishes

were performed in order to assess the direct implication of AKAP12 in controlling the phosphorylation of STAT3 Y705 upon removal of HDAC7 in HUVECs. Specific silencing of AKAP12 expression while HDAC7 is depleted decreases the phosphorylation of pSTAT3 on Y705 (Fig. 8c). In fact, the expression level of STAT3 pY705 returned to basal condition following HDAC7/AKAP12 codepletion. Notably, the sole depletion of AKAP12 abrogated the basal expression of the STAT3 pY705. This observation collectively indicates that AKAP12 is essential for the phosphorylation of STAT3 Y705 in HUVEC. Together with the previous findings (Fig. 6), these data point to the existence of a potential positive feedback loop between STAT3 and AKAP12 proteins.

\section{Discussion}

Considering the importance of angiogenesis in various pathologies, researchers and clinicians are involved in the the augmented levels of phosphorylated STAT3 Y705 and returns the levels of this protein to basal condition. Notably, suppression of AKAP12 alone further diminishes the basal levels of STAT3 pY705, leaving this protein form barely detectable in this condition. a-c The total levels of STAT3 as well as of the STAT3 pS727 are not affected (latter data not shown); representative images of one out of three independent biological replicates

ongoing quest to gain control over this process. The formation of new blood vessels from pre-existing vasculature depends on the expression of specific genes in endothelial cells, and accumulating evidence shows that the histone deacetylase family plays key role in the epigenetic regulation of these genes. Previously, we and others identified HDAC7 as one of key HDACs regulating angiogenesis $[4,5]$. However, the associated mechanism of action remains poorly understood.

Proteomic approaches integrating powerful techniques such as mass spectrometry are widely recognized as suitable tools for generating new hypotheses that are useful for providing the direction for more in-depth studies. Using this type of approach, we aimed to identify modulated proteins following siRNA-mediated depletion of HDAC7 in HUVECs and further the understanding of its control of angiogenesis. Among the generated data, the significant modulation of AKAP12 has deserved particular attention, as this protein displayed the highest and most reproducible 
up-regulation of all proteins identified. This protein has been previously described as a tumor-metastasis/-angiogenesis-suppressor gene. Indeed, forced expression of AKAP12 in prostate cancer cells suppressed a series of proangiogenic factors including VEGF, osteopontin, tenascin $\mathrm{C}, \mathrm{KGF}$, angiopoietin and HIF-1 $\alpha[9,20]$ leading to impaired angiogenesis and metastasis formation in vivo [10, 21-23]. A recent study demonstrated that treatment of endothelial cells with conditioned media from AKAP12expressing cancer cells blocks their abilities to migrate [24], sustaining the antiangiogenic role for AKAP12.

In this study, we demonstrated for the first time that depletion of HDAC7 in endothelial cells induces overexpression of AKAP12, which consequently leads to inhibition of their migration and blocks their abilities to form blood vessels in vitro. These findings indicate that increased expression of AKAP12 in endothelial cells exerts an anti-angiogenic effect per se. This observation may possibly be explained by the apparent interference of AKAP12 with the PI3K/AKT pathway, a pathway well described to regulate endothelial cell migration and angiogenesis. We previously demonstrated that HDAC7 depletion inhibited the phosphorylation of AKT. The current work demonstrates that this inhibition is partially relieved when HDAC7-depleted cells are cotransfected with the siRNA-targeting AKAP12 (Figure S5, Supplemental Data). Although it is not yet clear how AKAP12 might control the phosphorylation of AKT, the literature suggests that AKAP12 can scaffold phosphoinositides, leading to the uncoupling of the PI3K/AKT signaling cascade and interruption of AKT activation [12, 25]. However, it is also conceivable that AKAP12 could inhibit the phosphorylation of AKT via its ability to scaffold and modulate the activity of effector kinases such as PKC and PKA [26]. Further studies are needed to meticulously investigate the nature of the interaction between AKAP12 and AKT.

In the current study, we demonstrated for the first time that HDAC7 specifically controls histone $\mathrm{H} 3$ acetylation on the AKAP12 promoter. This is consistent with other studies showing that AKAP12 expression is associated with epigenetic modifications such as promoter hypermethylation [27-30]. However, the removal of HDAC7 is only a prerequisite for the transcriptional machinery to induce the gene expression. Accordingly, we sought to identify the transcription factor responsible for the induction of AKAP12 gene expression. Previously published ChIP studies pointed to the possible link between STAT3 and AKAP12 in the context of transcriptional regulation [15, 16]. We demonstrated that HDAC7 depletion in HUVECs induces a specific phosphorylation of STAT3 at the Y705 residue and found that this process is PKC dependent. Along these lines, we showed that this particular phosphorylated form of STAT3 is preferentially found in the nucleus, bound to the chromatin and AKAP12 promoter. STAT3 is required for the increased expression of AKAP12 in HDAC7-depleted HUVECs. Additionally, we hypothesized that STAT3 may further participate in regulating the expression of other angiogenesis-relevant genes. Therefore we have tested several proven STAT3 target genes, previously known as implicated in angiogenesis, and found that STAT3 is also modulating (up-regulating) the expression of ICAM1 upon removal of HDAC7 in HUVEC. ICAM1 is one of few key vascular adhesion molecules responsible for endothelial-immune cell interaction. In the tumor, excessive secretion of VEGF and basic fibroblast growth factor has been shown to down-regulate ICAM1 expression in associated endothelial cells [31]. This in turn results in the reduced ability of leukocytes to interact with the blood vessel endothelium and to infiltrate in the tumor [32]. Interestingly, previous studies [33] have demonstrated that epigenetic mechanisms are responsible for silencing of ICAM1 in tumor-conditioned endothelial cells and that global HDAC (trichostatin A) and DNA methyltransferase (5-aza-2-deoxycytidine and zebularine) inhibitors are able to restore ICAM1 expression. To this previous knowledge, the current study appends a clear mechanism where ICAM1 expression in endothelial cells is dependent of STAT3 activation, whereas HDAC7 serves as the corresponding transcriptional repressor.

Crosstalk between phosphorylation and acetylation is beginning to emerge as a crucial mechanism for regulating the functions of many transcription factors. Consistent with other studies [34-36], preliminary MS analysis and IP/WB experiments in the current study indicated that the depletion of HDAC7 induces an increase in the acetylation level of STAT3. (Figure S6, Supplemental Data). Therefore, the phosphorylation of STAT3 observed on Y705 could be triggered by acetylation of surrounding lysine residues and both PTM could actively interact to positively regulate STAT3 activity. Finally, we found that AKAP12 is also required for the phosphorylation of STAT3 on Y705. With the previous findings, these data suggest that a positive feedback loop may exist between AKAP12 and STAT3. Therefore, we hypothesize that the depletion of HDAC7 first induces acetylation of STAT3, which favors its phosphorylation. Activated STAT3 recruits an acetyltransferase, forming a complex that leads to the acetylation of histone $\mathrm{H} 3$ on the promoter of AKAP12. Consequently, this epigenetic modification allows for an increase in expression of AKAP12, which in turn participates in the phosphorylation of STAT3 Y705. This hypothetic model is schematized in Fig. 9 and merits further consideration in future studies.

In summary, the current study placed AKAP12 in the context of the HDAC7-mediated epigenetic control of 


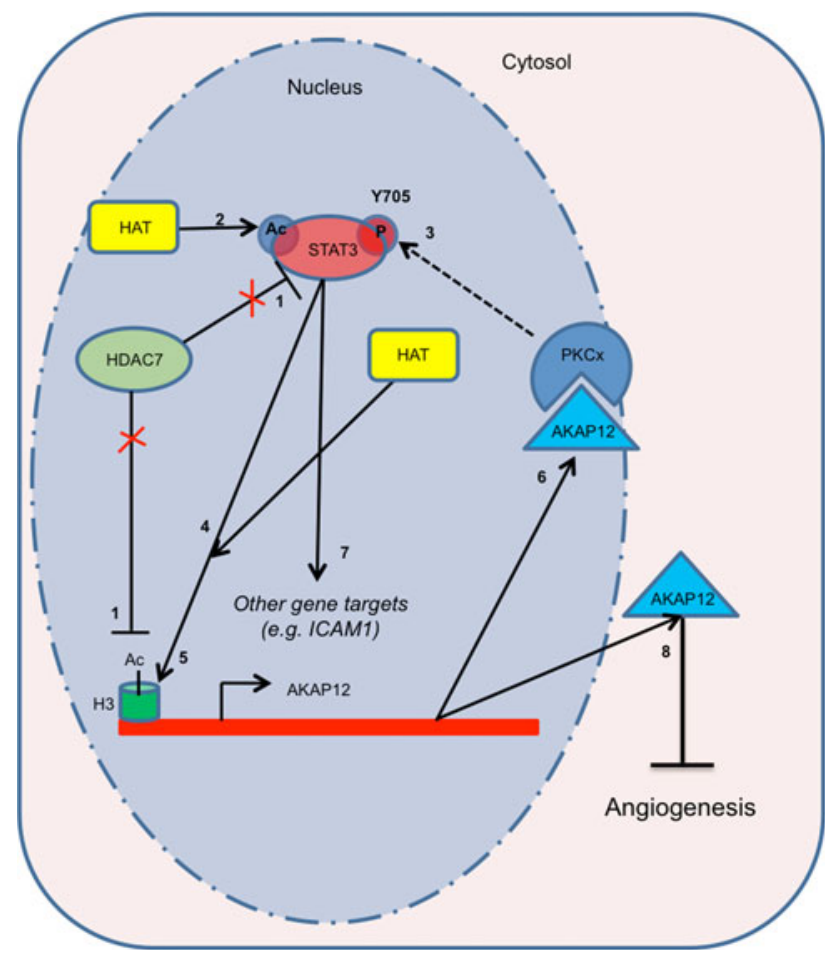

Fig. 9 The mechanistic model of HDAC7-mediated control of AKAP12 and angiogenesis in endothelial cells. AKAP12 is epigenetically regulated by HDAC7 (1), which in turn may also be involved in modulating the acetylation status of STAT3 (1). Removal of HDAC7 triggers STAT3 acetylation (2 [outlined in the Figure S6, Supplemental Data]) and PKC-dependent (3) phosphorylation of Y705 (dashed line indicates that an additional involvement of a tyrosine kinase is probably necessary). Activated STAT3 potentially recruits a histone acetyltransferase (4) leading to the acetylation of histone $\mathrm{H} 3$ on the promoter of AKAP12 gene (5). The event results in elevated levels of AKAP12, which takes part in multiple processes. It sustains the phosphorylation of STAT3, probably via its ability to scaffold PKCx (PKCx stands for the unknown isoform) (6). Activated STAT3 controls also other (angiogenesis) relevant genes like ICAM1 (7). Furthermore, AKAP12 targets other proteins (e.g. AKT, Figure S5, Supplemental Data) in order to suppress angiogenesis (8)

angiogenesis in endothelial cells and pointed to the need to consider such scaffolding proteins in this important process. There are remarkable similarities in the cellular and molecular mechanisms that are involved in both tumor invasion and angiogenesis [37]. Sustaining these observations we showed in this study that the tumor suppressor protein AKAP12 acts also as an angiogenesis suppressor. Further studies will focus more on the nature of the interaction between the AKAP12 scaffolding protein and its upand down-stream targets. These interactions may prove to be novel anchor points for directing the further development of antiangiogenic drugs.

Acknowledgments D. Mottet is a Research Associate and A. Bellahcène is a Senior Research Associate of the FNRS. N. Matheus is the FRIA Doctoral Fellow and B. Dumont is the Televie Doctoral Fellow of the FNRS. The authors acknowledge the GIGA-Proteomics
Platform of the ULg and Diagenode, Liege, Belgium for experimental support. The authors are particularly thankful to Prof. Irwin Gelman, Roswell Park Cancer Institute, USA, for his helpful discussions. This work was supported by grants from the Walloon Region (NEO-ANGIO), the University of Liège (Centre Anti-Cancéreux) as well as by FRIA and TELEVIE grants from the National Fund for Scientific Research (FNRS), Belgium.

Conflict of interest Authors have nothing to disclose and declare that there are no conflicts of interest.

\section{References}

1. Peserico A, Simone C (2011) Physical and functional HAT/ HDAC interplay regulates protein acetylation balance. J Biomed Biotechnol 2011:371832

2. Mottet D, Castronovo V (2010) Histone deacetylases: antiangiogenic targets in cancer therapy. Curr Cancer Drug Targets 10(8):898-913

3. Beumer JH, Tawbi H (2010) Role of histone deacetylases and their inhibitors in cancer biology and treatment. Curr Clin Pharmacol 5(3): 196-208

4. Mottet D et al (2007) Histone deacetylase 7 silencing alters endothelial cell migration, a key step in angiogenesis. Circ Res 101(12):1237-1246

5. Chang S et al (2006) Histone deacetylase 7 maintains vascular integrity by repressing matrix metalloproteinase 10 . Cell 126(2):321-334

6. Martin M et al (2008) Protein phosphatase 2A controls the activity of histone deacetylase 7 during $\mathrm{T}$ cell apoptosis and angiogenesis. Proc Natl Acad Sci USA 105(12):4727-4732

7. $\mathrm{Ha} \mathrm{CH}$ et al (2008) VEGF stimulates HDAC7 phosphorylation and cytoplasmic accumulation modulating matrix metalloproteinase expression and angiogenesis. Arterioscler Thromb Vasc Biol 28(10): 1782-1788

8. Wong W, Scott JD (2004) AKAP signalling complexes: focal points in space and time. Nat Rev Mol Cell Biol 5(12):959-970

9. Su B et al (2006) SSeCKS metastasis-suppressing activity in MatLyLu prostate cancer cells correlates with vascular endothelial growth factor inhibition. Cancer Res 66(11):5599-5607

10. Lee SW et al (2003) SSeCKS regulates angiogenesis and tight junction formation in blood-brain barrier. Nat Med 9(7):900-906

11. Su B et al (2010) SSeCKS/Gravin/AKAP12 inhibits cancer cell invasiveness and chemotaxis by suppressing a protein kinase CRaf/MEK/ERK pathway. J Biol Chem 285(7):4578-4586

12. Gelman IH (2010) Emerging roles for SSeCKS/Gravin/AKAP12 in the control of cell proliferation, cancer malignancy, and barriergenesis. Genes Cancer 1(11):1147-1156

13. Jaffe EA et al (1973) Culture of human endothelial cells derived from umbilical veins. Identification by morphologic and immunologic criteria. J Clin Investig 52(11):2745-2756

14. Deroanne CF, Lapiere CM, Nusgens BV (2001) In vitro tubulogenesis of endothelial cells by relaxation of the coupling extracellular matrix-cytoskeleton. Cardiovasc Res 49(3):647-658

15. Oh YM et al (2009) Prediction and experimental validation of novel STAT3 target genes in human cancer cells. PLoS ONE 4(9):e6911

16. Dauer DJ et al (2005) Stat3 regulates genes common to both wound healing and cancer. Oncogene 24(21):3397-3408

17. Pranada AL et al (2004) Real time analysis of STAT3 nucleocytoplasmic shuttling. J Biol Chem 279(15):15114-15123

18. Gartsbein M et al (2006) The role of protein kinase C delta activation and STAT3 Ser727 phosphorylation in insulin-induced keratinocyte proliferation. J Cell Sci 119(Pt 3):470-481 
19. Naviglio $S$ et al (2010) Leptin potentiates antiproliferative action of cAMP elevation via protein kinase A down-regulation in breast cancer cells. J Cell Physiol 225(3):801-809

20. Akakura S et al (2008) Loss of the SSeCKS/Gravin/AKAP12 gene results in prostatic hyperplasia. Cancer Res 68(13):5096-5103

21. Choi YK, Kim KW (2008) AKAP12 in astrocytes induces barrier functions in human endothelial cells through protein kinase Czeta. FEBS J 275(9):2338-2353

22. Choi YK et al (2007) AKAP12 regulates human blood-retinal barrier formation by downregulation of hypoxia-inducible factor1alpha. J Neurosci 27(16):4472-4481

23. Cheng $C$ et al (2007) Essential role of Src suppressed C kinase substrates in endothelial cell adhesion and spreading. Biochem Biophys Res Commun 358(1):342-348

24. Lee SW et al (2011) Inhibition of endothelial cell migration through the downregulation of MMP-9 by A-kinase anchoring protein 12. Mol Medicine Rep 4(1):145-149

25. Dimmeler $\mathrm{S}$ et al (1999) Activation of nitric oxide synthase in endothelial cells by Akt-dependent phosphorylation. Nature 399(6736):601-605

26. Li L et al (2006) Protein kinase C negatively regulates Akt activity and modifies UVC-induced apoptosis in mouse keratinocytes. J Biol Chem 281(6):3237-3243

27. Choi MC et al (2004) AKAP12/Gravin is inactivated by epigenetic mechanism in human gastric carcinoma and shows growth suppressor activity. Oncogene 23(42):7095-7103

28. Jo U et al (2006) AKAP12alpha is associated with promoter methylation in lung cancer. Cancer Res Treat 38(3):144-151
29. Tessema M et al (2008) Promoter methylation of genes in and around the candidate lung cancer susceptibility locus 6q23-25. Cancer Res 68(6): 1707-1714

30. Heller $\mathrm{G}$ et al (2008) Genome-wide transcriptional response to 5-aza-2'-deoxycytidine and trichostatin a in multiple myeloma cells. Cancer Res 68(1):44-54

31. Griffioen AW et al (1996) Endothelial intercellular adhesion molecule-1 expression is suppressed in human malignancies: the role of angiogenic factors. Cancer Res 56:1111-1117

32. Dirkx AE et al (2003) Tumor angiogenesis modulates leukocytevessel wall interac- tions in vivo by reducing endothelial adhesion molecule expression. Cancer Res 63:2322-2329

33. Hellebrekers DM et al (2006) Epigenetic regulation of tumor endothelial cell anergy: silencing of intercellular adhesion molecule-1 by histone modifications. Cancer Res 66(22):10770-10777

34. Xiao H et al (2003) Tip60 is a co-repressor for STAT3. J Biol Chem 278(13):11197-11204

35. Yuan ZL et al (2005) Stat3 dimerization regulated by reversible acetylation of a single lysine residue. Science 307(5707):269-273

36. Wang R, Cherukuri P, Luo J (2005) Activation of Stat3 sequencespecific DNA binding and transcription by p300/CREB-binding protein-mediated acetylation. J Biol Chem 280(12):11528-11534

37. Eccles SA (2004) Parallels in invasion and angiogenesis provide pivotal points for therapeutic intervention. Int $\mathrm{J}$ Dev Biol 48:583-598 